\title{
Preparation of Nanodimensional $C d S$ by Chemical Dipping Technique and their Characterization
}

\author{
Soumitra Patra, Partha Mitra*, Swapan Kumar Pradhan \\ Department of Physics, The University of Burdwan, 713104, Burdwan, India
}

Recived: May 18, 2010; Revised: February 18, 2011

\begin{abstract}
A simple and cost effective chemical technique has been utilized to grow cadmium sulphide $(C d S)$ nanoparticles at room temperature. The sample is characterized with XRD (X-ray diffractometer), SEM (Scanning electron microscope), TEM (Transmission electron microscope) and UV-VIS spectrophotometer. The particle size estimated using X-ray line broadening method is approximately $5 \mathrm{~nm}$. Instrumental broadening was taken into account while particle size estimation. The lattice strain was evaluated using Williamson-Hall equation. SEM illustrates formation of sub-micron size crystallites and TEM image gives particle size approximately between 4-5 nm. Optical absorption study exhibits a band gap energy value of about $2.6 \mathrm{eV}$.
\end{abstract}

Keywords: CdS thin film, chemical dipping, particle size, optical band gap

\section{Introduction}

The synthesis and characterization of cadmium sulphide via different techniques have attracted considerable attention due to their potential applications. Nanometer-sized semiconductor exhibit structural, electronic, optical, luminescence and photoconducting properties very different from their bulk properties ${ }^{1-2}$. Accordingly they have potential application prospects in solar cell, photodetector, laser, LED, high-density magnetic information storage and many others in semiconductor industries ${ }^{3}$. Cadmium sulphide (bulk band gap $2.4 \mathrm{eV}$ at $300 \mathrm{~K}$ ) has huge potential in this aspect ${ }^{3}$. The growth techniques of II-VI semiconductor nanoparticles are relatively cheap. The characteristic absorption of light for $\mathrm{CdS}$ is in the visible range. There are various methods to prepare CdS nanoparticles ${ }^{1,4-7}$. Some of the above-mentioned methods have some drawbacks. While some of the methods used unstable precursors, some requires high temperatures and some others are not cost effective ${ }^{1}$. In the presented work, a novel chemical dipping technique has been utilized to prepare nanopowders of $\mathrm{CdS}$ at room temperature. The technique involves successive dipping of a precleaned substrate in separately placed cationic and anionic precursor. Between every immersion it is rinsed in distilled water or ion exchanged water. The technique, often called SILAR (Successive ionic layer adsorption and reaction), is generally reported for preparation of metal sulphide thin films ${ }^{8-9}$. It has been utilized to grow CdS thin film by several researchers ${ }^{8,10}$. We have earlier reported preparation of $\mathrm{ZnS}$ and $\mathrm{SnS}$ thin films by SILAR ${ }^{11,12}$.

Normally under optimized deposition conditions, SILAR produces adherent thin films. The deposition parameters for getting adherent thin films include concentration and $\mathrm{pH}$ of the reacting baths, temperature of deposition and specific substrate treatment. In the present work, we have used SILAR technique to get nanocrystalline powders of $C d S$. The SILAR deposition from aqueous solutions is a very promising method because of its simplicity and economy. The aim of the present work was to promote interest in SILAR method as applied to preparation of nanocrystalline cadmium sulphide powders. The deposition parameters were adjusted to give a nonadherent thin film which could be scratched to give powder. Earlier we have reported preparation of $\mathrm{ZnS}$ and $\mathrm{SnS}$ nanopowders by this technique ${ }^{13}$. The structural and optical characterizations of the synthesized powders were carried out using X-ray powder diffractometer and UV-VIS spectrophotometer. Scanning electron microscopy (SEM) was used to illustrate the formation of crystallites and TEM was used for particle size estimation.

\section{Experimental}

Cadmium sulphide powder was synthesized by dipping a precleaned glass substrate in $0.1 \mathrm{M}$ cadmium chloride $\left(\mathrm{CdCl}_{2}\right)$ solution and $0.1 \mathrm{M}$ sodium sulphide $\left(\mathrm{Na}_{2} S\right)$ solution both kept at room temperature. The substrate was kept overnight in chromic acid and this was followed by rinsing in distilled water and ultrasonic cleaning in equivolume mixture of acetone and alcohol in an ultrasonic cleaner. Concentration of the reacting baths in excess of $0.125 \mathrm{M}$ leads to complete detachment of film from the substrate. Also concentration of reacting baths less than $0.075 \mathrm{M}$ gives extremely slow growth rate. Accordingly the concentration of $0.1 \mathrm{M}$ used in the experiment was found to be optimum to give a weakly adherent thin film and significant growth rate. The cadmium chloride bath, used for deposition of $C d S$ was prepared by adding $\mathrm{CdCl}_{2}$ (Merck) in deionized water. Similarly sodium sulphide flakes (Merck) was added in deionized water to prepare the $\mathrm{Na}_{2} \mathrm{~S}$ bath. The $\mathrm{pH}$ of $\mathrm{CdCl}_{2}$ was 4.1 and the $\mathrm{pH}$ of $\mathrm{Na}_{2} \mathrm{~S}$ was 11.7 respectively. The $\mathrm{pH}$ measurement was carried out in a Systronics $\mathrm{pH}$ meter (Model 335). Dipping of the substrate in cationic and anionic precursors leads to a very weakly adherent $C d S$ film that could be easily scratched from the substrate. The powder so obtained was thoroughly washed repeatedly in deionized water.

Dipping of the substrate in cationic precursors leads to the absorption of metal $\left(\mathrm{M}^{2+}\right)$ ions $\left[\mathrm{Cd}^{2+}\right.$ ion in this case $]$ and subsequent dipping in anionic precursors leads to the absorption of sulpher $\left(\mathrm{S}^{2-}\right)$ ions. The reaction on the substrate leading to the formation of metal sulphide can be represented as

$$
\mathrm{Cd}^{2+}+\mathrm{S}^{2-} \rightarrow \mathrm{CdS}
$$

One complete set of dipping involves dipping of the substrate in $\mathrm{CdCl}_{2}$ solution followed by dipping in $\mathrm{Na}_{2} \mathrm{~S}$ solution. Hundred (100) 
such dipping was performed in order to get sufficient amount of powder. X-ray diffraction (XRD) with $\mathrm{CuK}_{\alpha}$ radiation $(\lambda=1.5418 \AA$ ) was made for structural characterization of the synthesized powders. Absorption spectrum was recorded in UV-VIS spectrophotometer (Shimadzu, Model: UV-1800).

The crystallite size was calculated by the X-ray line broadening method using the Scherrer formula ${ }^{14,15}$ :

$$
D=\frac{k \lambda}{\beta \cos \theta}
$$

where $\lambda$ is the wave length of radiation used ( $\mathrm{CuK}_{\alpha}$ in this case), $k$ is the Scherrer constant, $\beta$ is the full width at half maximum (FWHM) intensity of the diffraction peak for which the particle size is to be calculated, $\theta$ is the diffraction angle of the concerned diffraction peak and $\mathrm{D}$ is the crystallite dimension (or particle size).

Normally for calculation of particle size, instrumental broadening is not taken into account ${ }^{15,16}$. In the present work, the instrumental broadening was taken into account. In Scherrer equation, $\beta$ represents the broadening due to particle size alone. In general the experimentally observed broadening $\left(\beta_{\mathrm{o}}\right)$ is the total contribution from particle size broadening $(\beta)$, instrumental broadening $\left(\beta_{\mathrm{i}}\right)$ and strain broadening $\left(\beta_{\mathrm{s}}\right)$ and is represented as ${ }^{14}$

$$
\beta_{o}=\beta+\beta_{i}+\beta_{s}
$$

Neglecting strain broadening we can write

$$
\beta=\beta_{o}-\beta_{i}
$$

The instrumental broadening arises from various factors such as non-parallelism of the incident X-ray beam; presence of other wavelengths apart from $\mathrm{CuK}_{\alpha}$ etc. and it is a constant for a particular experimental setup. Diffraction data from standard silicon $(\mathrm{S} i)$ powder was used to measure the instrumental broadening. The particle size is very high in the standard and the broadening is this case is due to instrument only. Figure 1 shows the XRD pattern of standard silicon powder. The peaks at $28.48,47.3,56.08,69.12,76.22,87.86,94.8$ and 106.56 correspond to those from standard silicon.

The lattice strain present in the sample was estimated using Williamson-Hall equation ${ }^{17}$

$$
\beta \cos \theta=\frac{k \lambda}{D}+2 \varepsilon \sin \theta
$$

where $\varepsilon$ is the lattice strain. In this method, $\beta \cos \theta(y)$ is plotted against $2 \sin \theta(x)$. The slope of the line gives the strain $(\varepsilon)$.

Band gap energy $\left(\mathrm{E}_{\mathrm{g}}\right)$ was derived from the mathematical treatment of the data obtained from the absorbance vs. wavelength with the following relationship ${ }^{18}$ :

$$
(\alpha h v)^{2}=\left(h v-E_{g}\right)^{n / 2}
$$

where $v$ is the frequency, $h$ is the Planck's constant, $n$ carries the value of either 1 or 4 depending on whether the material has direct or indirect band gap.

\section{Results and Discussion}

\subsection{Structural characterization}

Figure 2 shows the XRD patterns of the $C d S$ powder. The XRD was done after thorough washing of the synthesized powder in deionized water and heating the powder at $150{ }^{\circ} \mathrm{C}$ for 2 hours. The material was scanned in the range $15-90^{\circ}$. Intensity in arbitrary units is plotted against $2 \theta$ in Figure 1 . The peaks at $26.77^{\circ}, 43.97^{\circ}$ and $51.28^{\circ}$ are in good agreement with the Joint committee on powder diffraction standard (JCPDS) data belonging to cubic $C d S^{19}$. The corresponding reflecting planes are (111), (220) and (311) respectively. The observed broad hump (and therefore large FWHM) however suggests that the synthesized materials are nanocrystalline in nature with very small particle size.

Figure 3 shows the broadening (in FWHM) against $2 \theta$ obtained for standard silicon sample. A polynomial fitting of the experimentally observed values were made and are shown in Figure 3. The corresponding fitting equation (polynomial regression) is

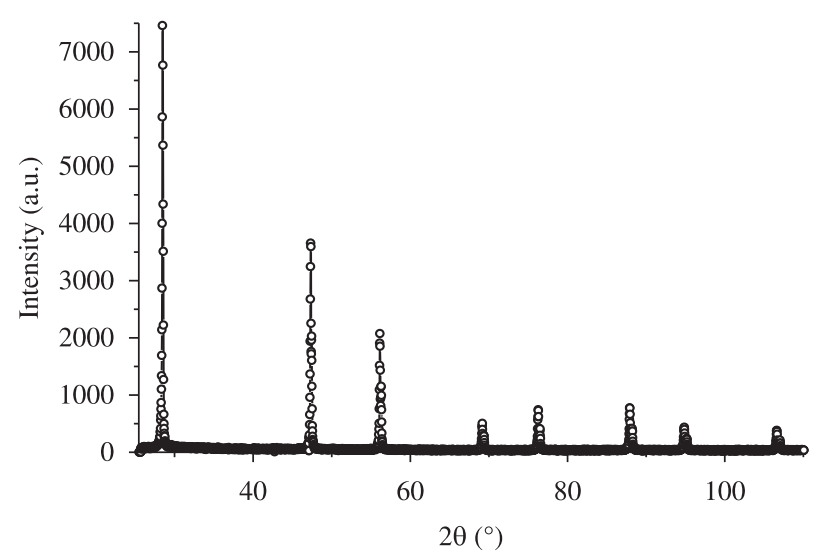

Figure 1. XRD pattern of standard silicon powder.

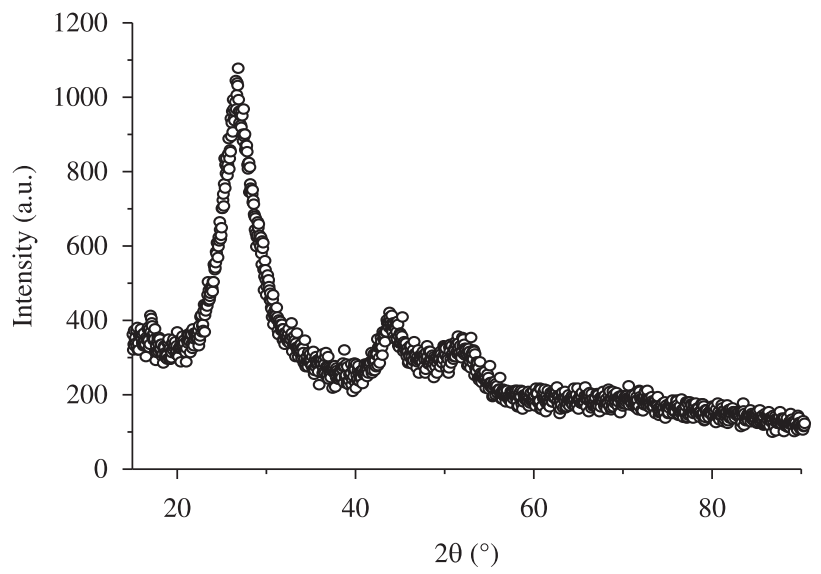

Figure 2. XRD pattern of $C d S$ powder.

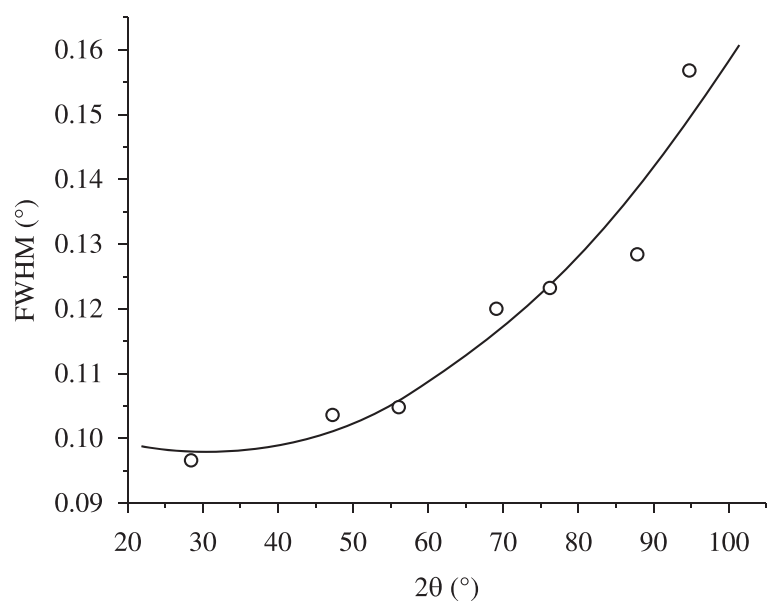

Figure 3. Instrumental broadening against $2 \theta$ for standard Silicon powder. 


$$
y=a+b x+c x^{2}
$$

where $y$ stands for the broadening in FWHM and $x$ is the angle in ${ }^{\circ} 2 \theta$. $a, b$ and $c$ are the constants with values $0.10977,-7.7418 \times 10^{-4}$ and $1.26278 \times 10^{-5}$ respectively.

Table 1 shows the values of $\beta_{o}, \beta_{i}$ and $\beta$ calculated using Equation 2. The instrumental broadening at the observed peak positions are calculated from Equation 3. The particle size calculated using Equation 1 is also shown in the table. The particle size was calculated using $k=0.9$, which corresponds to spherical crystallites and $\lambda=1.5418 \AA$, the wavelength of $\mathrm{CuK}_{\alpha}$ radiation. The average value of particle size taking instrumental broadening into account is $\sim 50.2 \AA$ (i.e. $\sim 5 \mathrm{~nm}$ ).

Table 1. Particle size for different peak positions considering instrumental broadening.

\begin{tabular}{ccccl}
\hline $\begin{array}{c}\text { Peak } \\
\text { position (20) }\end{array}$ & $\begin{array}{c}\beta_{\mathrm{o}} \\
\text { (in degrees) }\end{array}$ & $\begin{array}{c}\beta_{\mathrm{i}} \\
\text { (in degrees) }\end{array}$ & $\begin{array}{c}\beta \\
\text { (in degrees) }\end{array}$ & $\begin{array}{l}\text { Particle } \\
\text { size }(\AA)\end{array}$ \\
\hline 26.77 & 2.192 & 0.097 & 2.095 & 42.565 \\
43.97 & 1.720 & 0.099 & 1.621 & 68.36 \\
51.28 & 3.315 & 0.103 & 3.212 & 39.642 \\
\hline
\end{tabular}

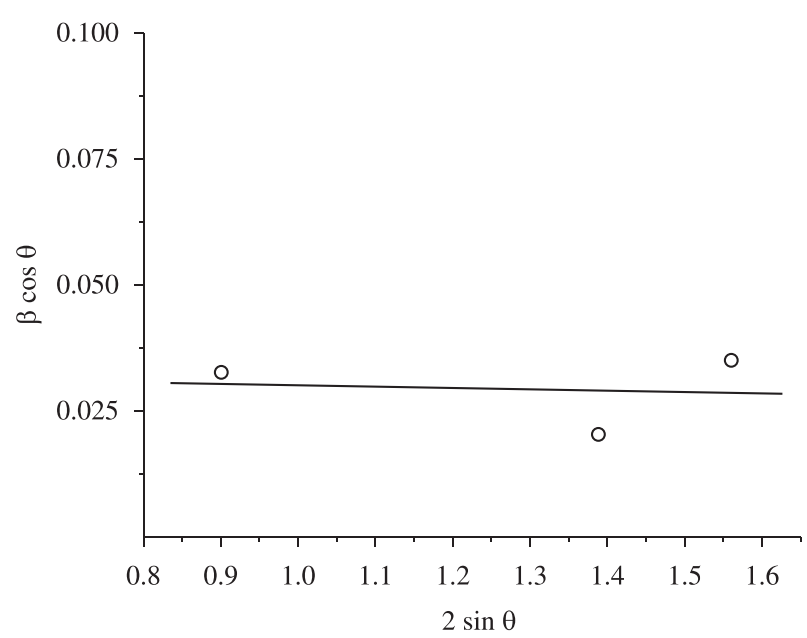

Figure 4. $\beta \cos \theta$ vs. $2 \sin \theta$ for $C d S$ nanocrystals.

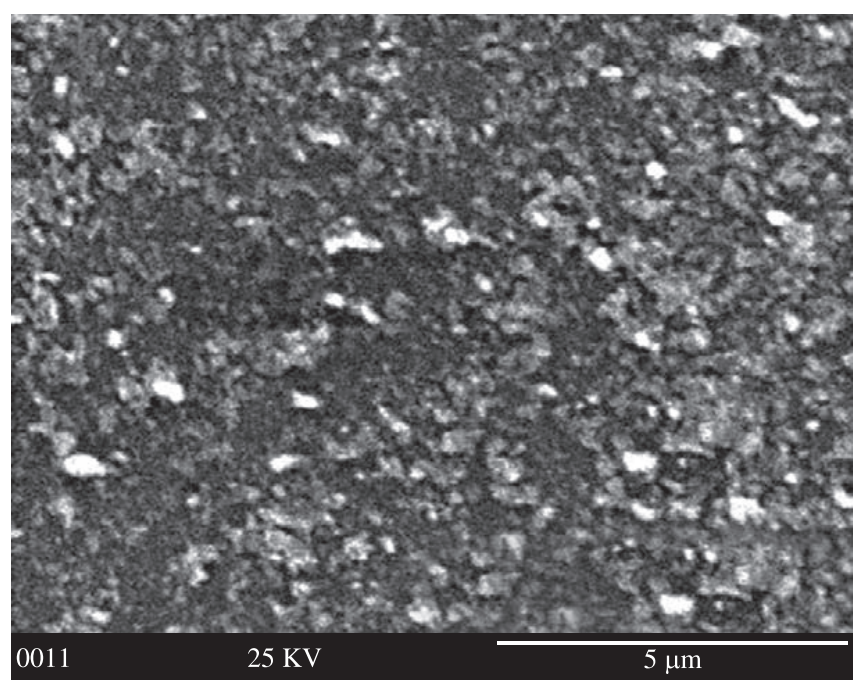

Figure 5. SEM image showing $C d S$ nanopowder.
Figure 4 shows the plot of $\beta \cos \theta$ against $2 \sin \theta$. The estimated slope and hence value of lattice strain is 0.0027 . The lattice strain introduced in the sample is possibly due to stacking fault within the crystallites. Since the deposition is carried out in ambient air, some stress is expected to be introduced which can result in stacking fault. The value of lattice strain is on the lower side compared to that reported (0.0494) by chemical precipitation technique ${ }^{20}$ possibly due to high particle size $(\sim 40 \mathrm{~nm})$ of the samples. Particle size corresponding to zero strain is $4 \mathrm{~nm}^{20}$. The calculation of lattice strain was done by Williamson-Hall method. A value of RMS strain in the range 0.001 to 0.003 has been reported for $\sim 10 \mathrm{~nm} C d S$ powder synthesized by ball milling ${ }^{21}$. Lattice strain was calculated by Rietveld's method.

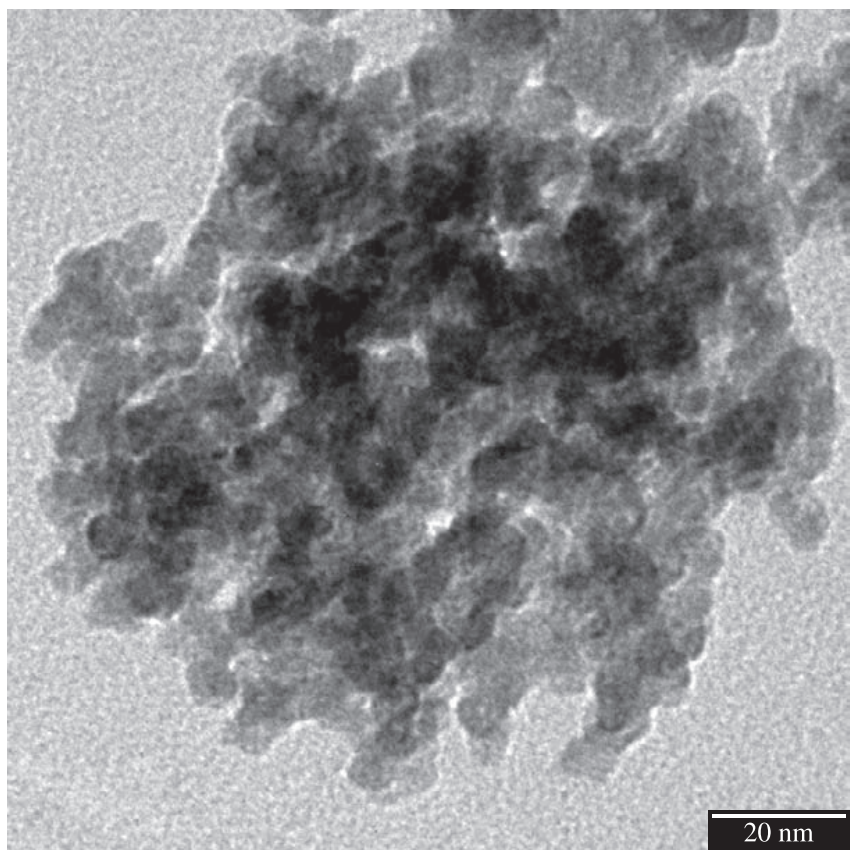

Figure 6. TEM image showing $C d S$ nanopowder.

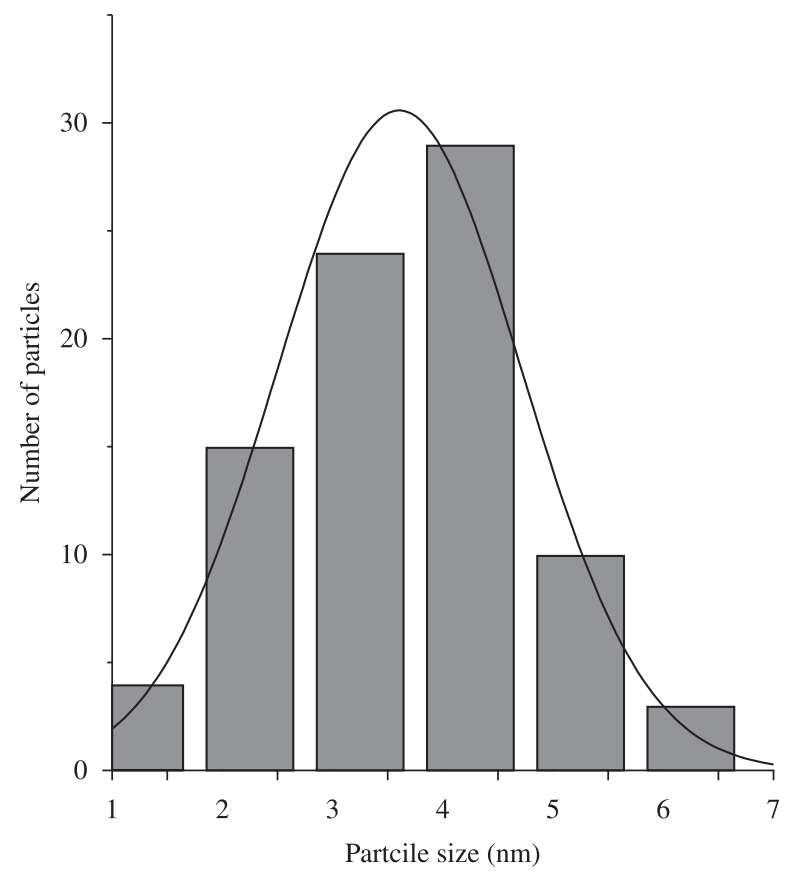

Figure 7. Histogram of partcile size distribution. 
Figure 5 shows the SEM image and Figure 6 shows the TEM image of $C d S$. The SEM image was taken before scratching the powder from the substrate. The SEM photograph clearly illustrates the formation of sub-micrometer crystallites distributed more or less uniformly over the surface. Agglomeration of small crystallites also seems to be present in certain regions on the surface. The particle size evaluated from TEM micrograph ranges approximately between 4-5 $\mathrm{nm}$. The histogram of particle size distribution is shown in Figure 7.

\subsection{Optical studies}

Absorption spectrum was recorded in a UV-VIS-NIR spectrophotometer (Shimadzu, Model UV-1800). Figure 8 shows the absorption spectrum of $C d S$ in the wavelength range 400-700 nm. Since $C d S$ has an indirect band gap, $\mathrm{n}=4$ was used and a plot of $(\alpha h v)^{2}$ as a function of $h v$ was drawn (Figure 9). Extrapolation of the line to the base line, where the value of $(\alpha h v)^{2}$ is zero, gives $\mathrm{E}_{\mathrm{g}}$ as $2.60 \mathrm{eV}$. This is on the higher side of that reported for $C d S^{3}$ indicating a shift due to quantum confinement arising from lowering of particle size. A bad gap of energy of $2.67 \mathrm{eV}$ has been reported for $C d S$ nanoparticles $\left(\sim 10 \mathrm{~nm}\right.$ particle size) by chemical reduction method ${ }^{22}$.

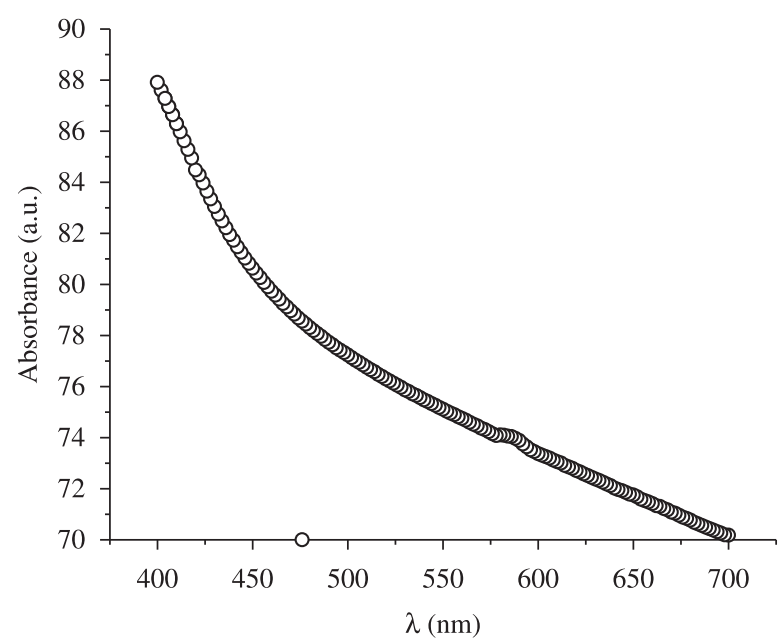

Figure 8. Optical absorption spectrum of $C d S$.

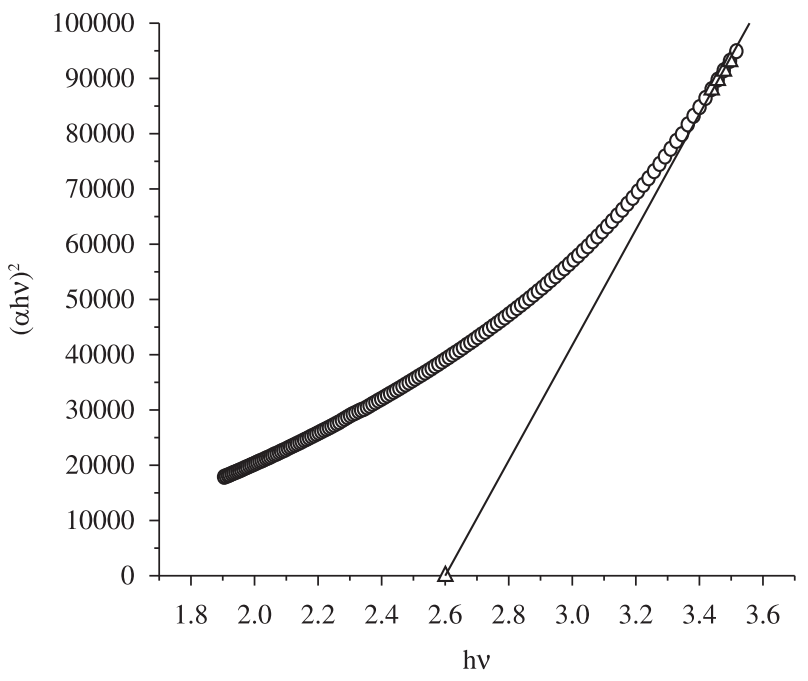

Figure 9. $(\alpha h v)^{2} v s . h v$ for $C d S$.

\section{Conclusions}

Nanocrystalline powders of $C d S$ can be prepared by SILAR technique. It seems that the technique can be extended for other metals sulphides as well. The technique is simple, cost effective and an easily scalable alternative to industrial level. The nanocrystalline powders so formed are extremely fine and their dimension is of the order $\sim 5 \mathrm{~nm}$ estimated using X-ray line broadening method. Average particle size obtained from TEM image ranges approximately between 4-5 $\mathrm{nm}$. Large shift in bandgap energy values compared to bulk values indicates quantum confinement. A detailed investigation of the materials synthesized under different conditions of precursor solutions is underway.

\section{References}

1. Bhattacharya R and Saha S. Growth of CdS nanoparticles by chemical method and its characterization. Pramana Journal of Physics. 2008; 71:187-192.

2. Peng XG, Manna L, Yang WD, Wickham J, Scher E, Kadavanich A et al. Shape control of CdSe nanocrystals. Nature. 2000; 404:59-61.

3. Tessler N, Medvedev V, Kazes M and Banin U. Efficient Near-Infrared Polymer Nanocrystal Light-Emitting Diodes. Science. 2002; 295:1506-1508.

4. Wang Y and Herron N. Nanometer-Sized semiconductor clusters: materials synthesis, quantum size effects, and photophysical properties. Journal of Physical Chemistry. 1991; 95:525-532.

5. Trindade T, O'Brien P and Zhang X. Synthesis of CdS and CdSe Nanocrystallites Using a Novel Single-Molecule Precursors Approach. Chemistry of Materials. 1997; 9:523-530.

6. Wu SH, Wu YS, Yang J, Han ZH, Xie Y, Qian YT et al. A novel solventothermal synthetic route to nanocrystalline $\mathrm{CdE}(\mathrm{E}=\mathrm{S}, \mathrm{Se}, \mathrm{Te})$ and morphological control. Chemistry of Materials. 1998; 9:2309-2312.

7. Li YD, Liao HW, Qian YT, Ding Y, Fan Y and Zhang Y. Solvothermal elemental direct reaction to $\mathrm{CdE}(\mathrm{E}=\mathrm{S}, \mathrm{Se}, \mathrm{Te})$ semiconductor nanorod. Inorganic Chemistry. 1999; 38:1382-1387.

8. Sun $\mathrm{H}$ and Mu J. silar deposition of cds thin films on glass substrates modified with 3-Mercaptopropyltrimethoxysilane. Journal of Dispersion Science and Technology. 2005; 26:719-722.

9. Sankapal BR, Mane RS and Lokhande CD. Successive ionic layer adsorption and reaction (SILAR) method for the deposition of large area $\left(\sim 10 \mathrm{~cm}^{2}\right)$ tin disulfide $\left(\mathrm{SnS}_{2}\right)$ thin films. Materials Research Bulletin. 2000; 35:2027-2035.

10. Sasagawa M and Nosaka Y. The effect of chelating reagents on the layer-bylayer formation of CdS films in the electroless and electrochemical deposition processes. Physical Chemistry Chemical Physics. 2001; 3:3371-3377.

11. Mitra $\mathrm{P}$ and Mondal S. Hydrogen and LPG sensing properties of $\mathrm{SnO}_{2}$ films obtained by direct oxidation of SILAR deposited SnS. Bulletin of Polish Academy of Sciences: Technical Sciences. 2008; 56:295-300.

12. Roy SD and Mitra P. Preparation of $\mathrm{ZnS}$ thin film by SILAR. Material Science Research India. 2008; 5:447-452.

13. Patra S, Mondal S and Mitra P. Preparation of ZnS and SnS nanopowders by modified SILAR technique. Journal of Physical Sciences. 2009; 13:229-234.

14. Klug $\mathrm{K}$ and Alexander L. X-ray diffraction procedures for polycrystalline and amorphous materials. New York: Wiley; 1974.

15. Chen M, Pei ZL, Sun C, Wen LS and Wang X. Formation of Al-doped ZnO films by dc magnetron reactive sputtering. Materials Letters. 2001; 48:194-198.

16. Prabhu R R and Khadar M A. Characterization of chemically synthesized CdS Nanoparticles. Pramana J. Phys. 2005; 65: 801-807.

17. Cullity BD, Stock SR. Elements of X-ray diffraction. $3^{\text {rd }}$ ed. Upper Saddle River: Prentice Hall; 2002.

18. Zainal Z, Nagalingam S and Hua TM. Properties of tin sulphide thin films electrodeposited in the presence of triethanolamine. Journal of Materials Science: Materials in Electronics. 2005; 16:281-285.

19. Joint Committee on Powder Diffraction Standards - JCPDS. International Centre for Diffraction Data. Swarthmore, PA; 1995. Card no.10-0454.

20. Singh V and Chauhan P. Structural and optical characterization of CdS nanoparticles prepared by chemical precipitation method. Journal of Physics and Chemistry of Solids. 2009; 70:1074-1079.

21. Patra S, Satpati B and Pradhan SK. Quickest Single-Step Mechanosynthesis of CdS quantum dots and their microstructure characterization. Journal of Nanoscience and Nanotechnology. 2011; 11:1-11.

22. Bhattacharya R and Saha S. Growth of CdS nanoparticles by chemical method and its characterization. Pramana Journal of Physics. 2008; 71:187-192. 